\title{
Ionic Conductivity and Dielectric Studies of Chitin Nanofiber (CNF) Incorporated PMMA Based Polymer Electrolytes
}

\author{
Shyly $\mathrm{PM}^{1}$, Karuppasamy $\mathrm{K}^{1}$, Linda $\mathrm{T}^{2}$, Paitip Thiravetyan ${ }^{3}$, \\ Balakumar $\mathrm{S}^{1}$ and Sahaya Shajan $\mathrm{X}^{1}$ \\ ${ }^{1}$ Center for Scientific and Applied Research (CSAR), School of Basic Engineering and Science, PSN College of \\ Engineering and Technology, Tirunelveli-627 152, Tamil Nadu, India. \\ ${ }^{2}$ Marthandam College of Engineering and Technology, Kuttakuzhi, Veeyannoor, Kanyakumari -629 177, Tamil \\ Nadu, India. \\ ${ }^{3}$ Division of Biotechnology School of Bioresearches and Technology, King Mongkut's University of Technology \\ Thonburi,(Bangkhuntien) 83 Moo 8 Thakham Bangkhuntien, Bangkok 10150, Thailand.
}

\begin{abstract}
Chitin nanofibers (CNF) are synthesized from shrimp cell chitin by stepwise purification and acid hydrolysis method. PMMA based polymer electrolytes complexed with $\mathrm{LIN}\left(\mathrm{CF}_{3} \mathrm{SO}_{2}\right)_{2}$ and chitin nanofibers as nanofiller have prepared by membrane hot-press technique. The prepared composite electrolytes are subjected to FT-IR, ac impedance, dielectric and modulus analyses. The incorporation of nanofiller in the polymer matrix increases the conductivity by an order of magnitude compared with filler free electrolytes. The complexation behavior of the composite polymer electrolytes are investigated by ATR FT -IR spectroscopy. The electric modulus analysis and dielectric studies of polymer electrolytes revealed that the non Debye dielectric relaxation nature.
\end{abstract}

Keywords: Chitin nanofiber, ac impedance, dielectric studies, electric modulus analysis

\section{Introduction}

The development of electrochemical devices such as batteries, super capacitors, electro-chromic devices and sensors has increased rapidly in the past few decades. Enormous interest has been shown in the development of rechargeable batteries, especially in acquiring a battery that combines long life, environmental safety, compact shape, low cost and high energy density. Lithium ion batteries appear as one of the most promising power sources due to their high energy densities compared to lead-acid and nickel-cadmium batteries [1-3]. Polymer electrolytes exhibit several interesting properties like flexibility, transparency, light weight and feasibility of thin film formation. It finds applications in variety of fields such as rechargeable batteries, fuel cells, sensors, aerospace, automobile and electronic industries. Among the various polymer electrolytes, composite solid polymer electrolytes offers many advantages, viz. solid-state non-corrosive medium, high automation potential for electrode preparation and cell and assembly techniques, broad operating temperature range, no separator, no filling procedure, intrinsic safety, etc [4]. Much attention is focused on investigating the polymer electrolytes to enhance the ambient temperature conductivity by blending of polymers, cross-linking, and incorporation of ceramic fillers or plasticization [5]. Though the addition of plasticizer or blending with other polymer leads to increase the conductivity to significant extent but it deteriorates the strength of film. In order to obtain a better polymer electrolyte with good conducting property and appropriate mechanical strength, a suitable nanofiller is embedded into the polymer matrix. The nanofiller can tend to suppress the crystalline nature (amorphous) of polymer thereby increasing the ionic conductivity [6].

PMMA is a transparent polymeric material that posses many desirable properties such as light weight, high light transmittance, chemical resistance, uncolored, resistance to weathering corrosion and good insulating properties [7]. PMMA has been used as a polymer host due to its high stability at the lithium-electrolyte surface and because it is less reactive towards the lithium electrode. Moreover, MMA (methyl methacrylate) monomer in PMMA has a polar functional group in the main polymer chain that has a high affinity for lithium ions, which are transported. Oxygen atoms from the MMA structure will form a coordinate bond with the lithium ion from doping salts. Hence the increase in effective ionic transport of PMMA based polymer electrolytes may due to the presence of polar group functional group in PMMA.

In the present work, chitin nanofiber (CNF) is employed as nanofiller. The natural linear amino polysaccharide Chitin (N-acetyl- $\beta$-D-glucosamine) possesses several interesting properties such as biocompatibility, bioactive, antimicrobial activity, low toxicity and ecological friendly. It finds applications in drug delivery system, paper finishing, solid state batteries, food industries and tissue engineering [8]. Lifts (Lithium bis $\sim$ trifluoromethanesulfonylimide $\left(\mathrm{LiN}\left(\mathrm{CF}_{3} \mathrm{SO}_{2}\right)_{2}\right)$ is an appealing salt since it decreases the polymer host's crystallinity and is more thermally and chemically stable than other traditional lithium salts [9]. 


\subsection{Materials}

\section{Materials, Methods and Characterization}

Poly (methyl methacrylate) with an average $M_{\mathrm{w}}=35,0000$ was obtained from Sigma-Aldrich. Lithium bis (trifluoromethanesulfonylimide) salt, LiTFSI $\left[\mathrm{LIN}\left(\mathrm{CF}_{3} \mathrm{SO}_{2}\right)_{2}\right]$ was obtained from Merck and dried at $100{ }^{\circ} \mathrm{C}$ for 1 hour to eliminate trace amounts of water in the material, prior to the preparation of composite polymer electrolytes. Chitin nanofiber (CNF) was synthesized from shrimp cell as the method reported earlier [10]. THF obtained from Merck was used as common solvent without further distillation.

\subsection{Preparation of polymer composite membrane}

The appropriate weight contents of PMMA, CNF and LiTFSI were dissolved in tetrahydrofuran(THF). The solution was then stirred continuously until the mixture took a homogeneous viscous liquid appearance. The resulting solution was poured on to a Teflon pushes and the THF was allowed to evaporate in air at room temperature. Then the samples were hot pressed into film specimens at high temperature. The thickness of the film was measured by means of a micrometer screw gauge. Different specimen of composite membranes was prepared by varying the amount of polymer and filler. The various compositions of filler, lithium salt and polymer were tabulated in Table 1 . The homogeneous, self standing polymer electrolyte films obtained from hot-press method were stored in vacuum desiccators and then subjected to further characterizations.

\subsection{Characterization techniques}

The impedance spectroscopy of the membranes was carried out by measuring in the $100 \mathrm{mHz}$ to $100 \mathrm{kHz}$ frequency range using electrochemical impedance analyzer (Zahner IM6, Germany) connected to a computer for data acquisition. The polymer membranes were kept in a gold plated solid sample holder mounted inside a vacuum cylindrical glass container with signal amplitude of $1 \mathrm{~V}$ at 16 points per frequency decade from room temperature to $423 \mathrm{~K}$ over wide frequencies range from $100 \mathrm{mHz}$ to $100 \mathrm{kHz}$. The dielectric and modulus spectroscopic analysis analyses were studied using (Zahner IM6, Germany) electrochemical analyzer for temperatures varying from room temperature to $423 \mathrm{~K}$ over wide frequencies range between $100 \mathrm{mHz}$ and 100 kHz. The ATR FT-IR spectra of polymer electrolytes were recorded using JASCO LT /FTIR 4100 (Japan) spectrophotometer in the region $400-4000 \mathrm{~cm}^{-1}$ with a signal resolution of $8 \mathrm{~cm}^{-1}$. The whole experiments were recorded in transmittance mode.

\begin{tabular}{|c|c|c|c|}
\hline Samples & PMMA (Wt\%) & $\mathrm{LiN}\left(\mathrm{CF}_{3} \mathrm{SO}_{2}\right)_{2}(\mathrm{Wt} \%)$ & $\mathrm{CNF}(\mathrm{Wt} \%)$ \\
\hline F1 & 95 & 5 & ---- \\
F2 & 90 & 5 & 5 \\
F3 & 85 & 5 & 10 \\
F4 & 80 & 5 & 15 \\
\hline
\end{tabular}

Table 1: Composition of PMMA, $\mathrm{LiN}\left(\mathrm{CF}_{3} \mathrm{SO}_{2}\right)_{2}$ and $\mathrm{CNF}$

\subsection{Ionic Conductivity}

\section{Results and Discussions:}

Ionic conduction in polymer electrolytes is due to the migration of lithium ions in the free volume of polymer matrix. The Arrhenius plot of variation of ionic conductivity as a function of reciprocal of temperature for polymer electrolytes containing different weight contents of filler and polymer are shown in fig.1. The ionic conductivity of the CNF incorporated polymer electrolytes are determined by means of following expression

$$
\sigma={ }^{t} / R_{b} A
$$

where $t$ and $A$ are thickness and area of cross section of the polymer electrolytes. $R_{b}$ represents bulk resistance which is obtained from extrapolation of semicircular region to highest frequencies.

It is observed from the figure that the conductivity of the polymer electrolyte increases with increase in temperature. i.e., it obeys Vogel-Tamman-Fulcher (VTF) relation

$$
\sigma=A T^{1 / 2} e^{-B_{R}-T_{g}}
$$

where $T_{g}$ is the temperature at which the segmental mobility of the polymer backbone ceases to exist and is generally the same as the glass transition temperature. A and B are constants. The constants A in VTF relation is related to number of charge carriers and B related to activation energy of the ion transport associated with configurational entropy of the polymer electrolyte [11]. It is also noticed from the figure that the conductivity increases with increase in CNF content up to $10 \mathrm{wt} \%$. This is due to the fact that the CNF increases the polymer chain segmental motion as well as amorphous region. These amorphous region favours the migration of lithium ion in the free volume of polymer matrix. These results are in agreement with the results reported earlier [12]. The conductivity of CNF incorporated PMMA based polymer electrolytes increases upon one order of magnitude compared to filler free electrolyte F1. In the mean time, the incorporation of CNF increases beyond $10 \mathrm{wt} \%$, the sudden decrease in ionic conductivity have observed which is due to phase 
discontinuities and dilution effect predominates in the polymer matrix. Also the addition of lithium salt (LiTFSI) in the polymer is optimized and the optimized wt $\%$ is 5 . When the concentration of LiTFSI exceeds $5 \mathrm{wt} \%$, the sample with anion $\left[\left(\mathrm{CF}_{3} \mathrm{SO}_{2}\right)_{2}\right]^{2-}$ forms neutral ion pairs with cation which leads to decrease the available number of free moving ions (charge carriers) in the polymer matrix. The maximum ionic conductivity is found to be in the range of $1.34 \times 10^{-5.2} \mathrm{~S} / \mathrm{cm}$ for polymer electrolyte $\mathrm{F} 3$ (10 wt\% CNF). These results are in close agreement with the result reported earlier by Stephan et al., using PEO as polymer host [13].

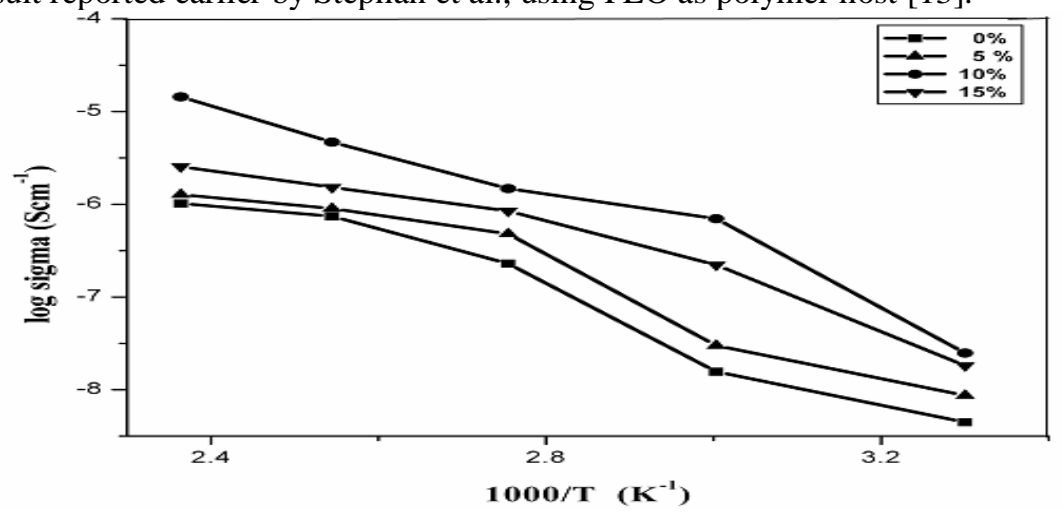

Fig.1. Arrhenius plot of log of ionic conductivity as a function of reciprocal of temperature for various prepared polymer electrolytes.

\subsection{Dielectric Spectral analysis}

The amount of charge that can be stored by polymeric material is evaluated by means of dielectric spectral analysis. In order to confirm the enhancement of ionic conductivity is due to an increase in available number of mobile charge carriers, dielectric study is performed. The dielectric permittivity of polymer electrolyte is determined by means of following equation

$$
\varepsilon^{*}=\varepsilon^{r}-j \varepsilon^{\mathrm{m}}
$$

where $\varepsilon^{\prime}$ and $\varepsilon^{\prime \prime}$ denotes the dielectric constant and dielectric loss of the system.

$$
\varepsilon^{\mathrm{m}}=\sigma^{\mathrm{r} /} /\left(\omega \varepsilon_{0}\right)
$$

$\sigma^{\prime}$ implies the real part of conductivity, $\omega$ the angular frequency and $\varepsilon_{0}$ is the permittivity of free space.

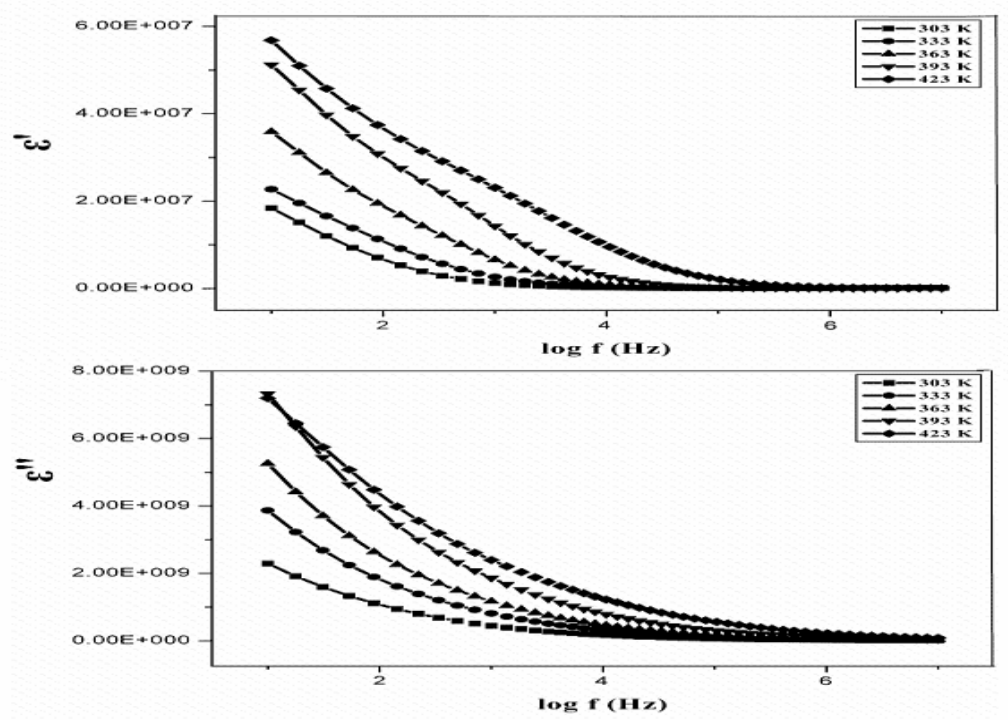

Fig.2. (a) Frequency dependence of dielectric constant $\varepsilon^{\prime}$ at various temperatures for F3.

$\&$

(b) Frequency dependence of dielectric loss $\varepsilon$ " at various temperatures for F3.

Fig.2 (a) shows the dielectric constant as a function of logarithmic frequency in the temperature range between $303 \mathrm{~K}$ and $423 \mathrm{~K}$ using $\mathrm{CNF}$ as nanofiller for polymer electrolyte $\mathrm{F}$. The purpose of choosing electrolyte sample F3 is due the fact that it yields maximum conductivity at room temperature. It is observed from the figure that $\varepsilon^{\prime}$ value increases sharply at lower frequencies region. The increase in $\varepsilon^{\prime}$ at lower frequencies is due to enhancement of charge carrier density in the space charge accumulation region which is also known as nonDebye type of behavior [14]. Also at lower frequencies the contribution of charge carriers increases towards 
dielectric constant. This results in an increase in equivalent capacitance at the electrolyte interface. In the mean time, $\varepsilon^{\prime}$ decreases at higher frequencies due to the high periodic reversal of the applied electric field at the electrolyte-electrode interface, so that there is no excess ion diffusion in the direction of the electric field. It is also evident from the figure, as the temperature increases the value of dielectric constant also increases. This is because of the reason that the available number of free moving ions/charge carriers increases at high temperature.

Fig.2 (b) shows the variation of dielectric loss $\left(\varepsilon^{\prime \prime}\right)$ as a function of logarithmic frequency for polymer electrolyte $\mathrm{F} 3$ at different temperatures. It is observed from the figure that the dielectric loss increases with increase in temperature and decrease in frequency is due to formation of free charges at the electrolyte-electrode interface. At high frequencies dielectric loss decreases owing to the reason of reduction of charge carriers at the interface between electrode and electrolyte.

\subsection{Electric modulus analysis}

Complex modulus electric spectra analysis is used to examine the electric response of PNCSPE. The frequency dependent electric modulus $M^{*}(\omega)$ is expressed by means of following equation

Where $M^{\prime}(\omega)$ and $M^{\prime \prime}(\omega)$ represents real part of electric modulus and imaginary part of electric modulus

i.e.,

where $\mathrm{C}_{\mathrm{o}}$ is the vacuum capacitance of the cell and $\varepsilon^{\prime}(\omega)$ is the complex permittivity and $\mathrm{j}=\sqrt{ }-1$ respectively.
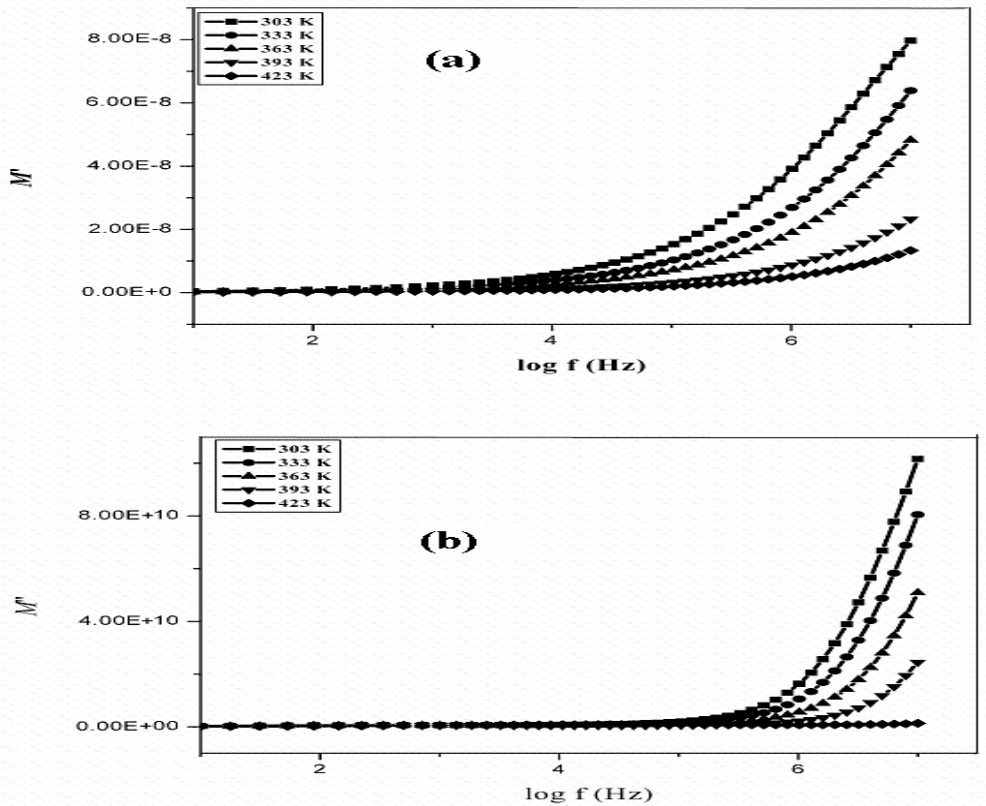

Fig.3. (a) Frequency dependence of real part of modulus spectra M' at various temperature for F3. (b) Frequency dependence of imaginary part of modulus spectra M' at various temperature for F3.

Fig.3 (a \& b) shows the frequency dependence of real $\left(M^{\prime}\right)$ and imaginary $\left(M^{\prime \prime}\right)$ modulus formalism for sample F3 at different temperature. At higher frequencies, $M^{\prime} \& M^{\prime \prime}$ increases gradually with a tendency for saturation. The observed dispersion is mainly due to conductivity relaxation spread over range of frequencies. At higher temperature, the peak shift to higher frequencies. As the temperature increase, the peaks of $M^{\prime}$ and $M^{\prime \prime}$ have decreased gradually due to plurality of relaxation mechanism. The value of $M^{\prime}$ and $M^{\prime \prime}$ tends to be zero in the vicinity at lower frequencies which proposes that the suppression of the electrode polarization at interface is negligible at lower frequencies. The presence of long straight line in the low frequency region confirms a large equivalent capacitance associated with electrode interface. In the mean time the value of $M^{\prime}$ and $M^{\prime \prime}$ decreased slowly at higher temperature due to decrease in charge carrier density at the space accumulation region. These outcomes are in accordance with the results reported by literature earlier [15].

\subsection{FT-IR analysis}

The FT-IR spectra of pure PMMA, LiTFSI, 95\%PMMA+5\%LiTFSI (F1), CNF and 85\% PMMA+5\%LiTFSI+10\%CNF (F3) are shown in fig.4 panel (a-e). In fig.1.panel (a), the characteristic 
Ionic conductivity and Dielectric studies of Chitin nanofiber (CNF) incorporated PMMA based bands of pure PMMA that appeared at 1460, 1390, 960 and $1762 \mathrm{~cm}^{-1}$ corresponds to $\mathrm{CH}_{3}$ stretching, $\mathrm{CH}_{2}$ twisting, C-O-C and $\mathrm{C}=\mathrm{O}$ stretching respectively. Panel (b) represents the FT-IR spectrum of $\mathrm{LiN}\left(\mathrm{CF}_{3}\left(\mathrm{SO}_{2}\right)_{2}\right)$. The vibration peaks that observes at 1145 and $1340 \mathrm{~cm}^{-1}$ corresponds to $\mathrm{C}-\mathrm{F}$ stretching and $\mathrm{S}=\mathrm{O}$ asymmetric stretching of $\operatorname{LiN}\left(\mathrm{CF}_{3}\left(\mathrm{SO}_{2}\right)_{2}\right)$. Panel (c) clearly demonstrates the interaction between salt and polymer. The peaks at 1390, 960 and 1762 are shifter to 1395,967 and $1768 \mathrm{~cm}^{-1}$ during complexation with lithium salt. The peaks of $1656 \mathrm{~cm}^{-1}$ (amide I: C=O), $1621 \mathrm{~cm}^{-1}$ (amide II: $\mathrm{NH}_{2}$ ), $1557 \mathrm{~cm}^{-1}(-\mathrm{CNH}), 3437 \mathrm{~cm}^{-1}$ (amine: $\mathrm{N}-\mathrm{H}$ ) represents CNF polymer. The peaks of hydroxyl group $(\mathrm{OH})$ from $\mathrm{CNF}$ were obtained from the peak of 1260 , $1312,1378,2890$ and $3261 \mathrm{~cm}^{-1}$ as shown in panel (d). The incorporation of CNF made appropriate changes in the FT-IR spectrum of polymer electrolyte. The shifting of peaks of CNF from 2890,890 and $3261 \mathrm{~cm}^{-1}$ into 2898, 896 and $3271 \mathrm{~cm}^{-1}$ during complexation. It is also evident from panel (e) the change in shape of $\mathrm{CH}_{3}$ stretching mode of PMMA from single peak to double peaks which indicates the presence of coordination of $\mathrm{CH}_{3}$ bond with CNF. Hence FT-IR spectrum is a powerful tool to identify the complexation behavior and structural changes in polymer electrolytes.

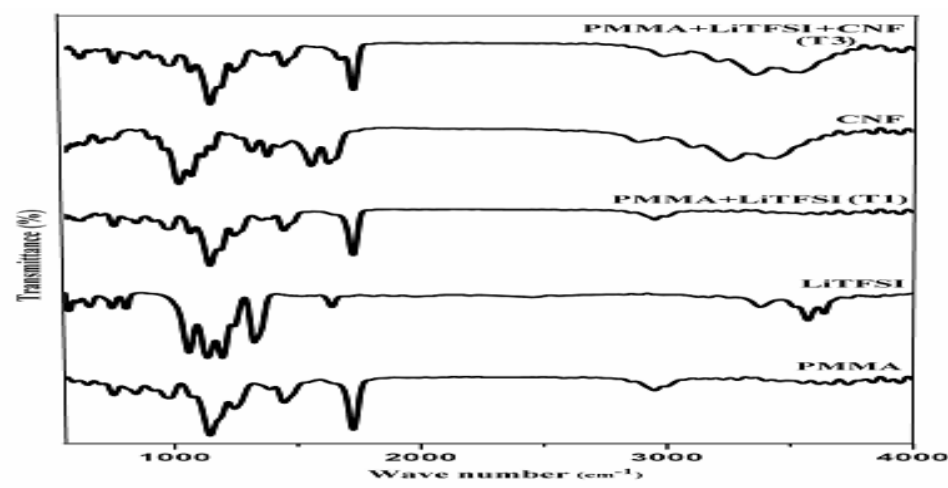

Fig.4. FT-IR spectra of (a) Pure PMMA, (b) $\mathrm{LiN}\left(\left(\mathrm{CF}_{3} \mathrm{SO}_{2}\right)_{2}\right)$, (c) F1, (d) CNF and (e) F3.

\section{Conclusions}

Various series of PMMA based polymer nanocomposite electrolytes containing $\mathrm{LiN}\left(\left(\mathrm{CF}_{3} \mathrm{SO}_{2}\right)_{2}\right)$ as salt and $\mathrm{CNF}$ as nanofiller were prepared by hot-press technique and then subjected to various electrochemical characterizations. The maximum ionic conductivity was found to be in the range of $10^{-5.2} \mathrm{~S} / \mathrm{cm}$ for electrolyte containing $85 \%$ PMMA+5\% $\mathrm{LiN}\left(\left(\mathrm{CF}_{3} \mathrm{SO}_{2}\right)_{2}\right)+10 \% \mathrm{CNF}$. The low dispersion of $\varepsilon$ ' and $\varepsilon$ " revealed that the space charge effects happening from the electrodes. The structural and conformational changes in the polymer host due to entrapment of nanoparticles and ion-polymer interaction were investigated by ATR FT-IR analysis.

\section{References}

[1] J.R.Maccallum, C.A.Vincent (Eds.), Polymer electrolyte reviews (London, Elsevier Vol. I, 1989).

[2] F.M.Gray, Solid polymer electrolytes-Fundamentals and Technical applications (Germany, ACH, Weinheim, 1991).

[3] P.G. Bruce, Solid state electrochemistry (Cambridge, Cambridge University Press, 1995).

[4] A.Manuel Stephan, Review on gel polymer electrolytes for lithium batteries, Eur. Polym. J. 42, (2006) 21-42

[5] A. Manuel Stephan, K.S.Nahm, T.Premkumar, M.Anbukulanthainathan, G.Ravi and J.Wilson, Nanofiller incorporated poly(vinylidene fluoride-hexafluoropropylene) (PVdF-HFP) composite electrolytesfor lithium batteries, J. Power Sources., 159, (2006) 1316-1321.

[6] H. H. Sumathipala, J. Hassoun, S. Panero and B. Scrosati, High performance PEO-based polymer electrolytes and their application in rechargeable lithium polymer batteries, Ionics, 13, (2007) 281-286.

[7] S.Ramesh and Liew Chiam Wen, Investigation on the effects of addition of $\mathrm{SiO}_{2}$ nanoparticles on ionic conductivity, FT-IR and thermal properties of nanocomposite PMMA-LiCF $3 \mathrm{SO}_{3}-\mathrm{SiO}_{2}$, Ionics, 16, (2010), 255-262.

[8] P.M.Visakh and Sabu Thomas, Preparation of bionanomaterials and their polymer nanocomposites from waste and biomass, Waste Biomass Valor, 1, (2010) 121-134.

[9] K. Kanamura, T. Umegaki, S. Shriraishi, M. Ohashi, and Z. Takehara, Attenuation of aluminum current collector corrosion in LiTFSI electrolytes using fumed silica nanoparticles, J. Electrochem.Soc, 149, (2004) A228-A230.

[10] Rujira Dolphen and Paitip Thiravetyan, Adsorption of melanoidins by chitin nanofibers, Chem. Eng.J. 166, (2011) 890-895.

[11] K.M. Abraham, Z.Jiang and B.Carroll, Highly conductive PEO-like polymer electrolytes, Chem. Mater. 9, (1997) 1978-1988.

[12] S.Ramesh and G.P.Ang, Impedance and FT-IR studies on plasticized PMMA-LiN(CF3SO2)2 nanocomposite polymer electrolytes, Ionics, 16, (2010) 465-473.

[13] N Angulakshmi, T Premkumar, Sabu Thomas and A.Manuel Stephan, Ionic conductivity and interfacial properties of nanochitinincorporated polyethylene oxide- $\mathrm{LiN}\left(\mathrm{C}_{2} \mathrm{~F}_{5} \mathrm{SO}_{2}\right)_{2}$ polymer electrolytes, Electrochim.Acta, 55, (2010) 1401-1406.

[14] Hellar Nithya, S.Selvasekarapandian, P.Cristopher Selvin, D. Arunkumar and Muthusamy Hema, Structural and ionic conductivity studies on P(ECH-EO): $\gamma$-BL:LiClO4 plasticized polymer electrolytes, Ionics, 17, (2011) 587-593.

[15] K.S. Yap, L.P. Teo, L.N. Sim, S.R. Majid and A.K. Arof, Investigation on dielectric relaxation of PMMA-grafted natural rubber incorporated with $\mathrm{LiCF}_{3} \mathrm{SO}_{3}$, Physica B, 407, (2012) 2421-2428. 